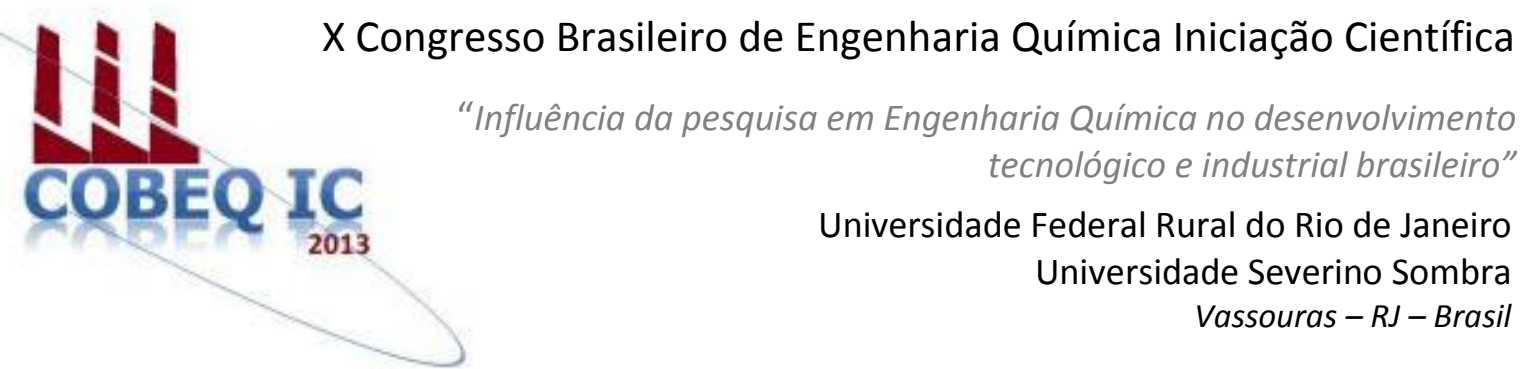

\title{
INFLUÊNCIA DA CONCENTRAÇÃO LIPÍDICA NA SECAGEM DE PASTA DE VEGETAIS ENRIQUECIDA COM SANGUE BOVINO EM LEITO DE JORRO
}

\author{
RODRIGUES $^{1^{*}}$, M. C. K.; DA ROCHA ${ }^{1^{*}}$, S. F.; MONTE ${ }^{1^{*}}$, M. L.; LARROSA ${ }^{2^{*}}$, A. P. Q.; \\ PINTO $^{3}$, L. A. A. \\ ${ }^{1}$ Alunas da EQA/FURG; ${ }^{2}$ Doutoranda - EQA/FURG; ${ }^{3}$ Docente - EQA/FURG \\ Escola de Química e Alimentos - Laboratório de Operações Unitárias - FURG, \\ Endereço - FURG- Rua Alfredo Huch, 475, Rio Grande, CEP 96.201-900, RS \\ email: dqmpinto@furg.br
}

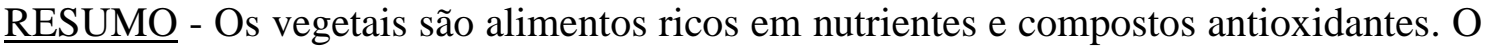
sangue bovino é um subproduto de frigoríficos, rico em proteínas. A secagem em leito de jorro apresenta vantagens por se tratar de um equipamento de baixo custo operacional, obtendo um produto de qualidade similar ao spray dryer. No entanto, estudos têm demonstrado que a composição química de pastas alimentícias na secagem em leito de jorro pode interferir no desempenho do equipamento. Diante disso, este trabalho teve como objetivo avaliar diferentes concentrações lipídicas a fim de melhorar o desempenho da secagem em leito de jorro de pasta de vegetais enriquecida com sangue bovino. A pasta foi formulada fixando as concentrações de lipídios em 4 e 6\%, obtendo duas formulações diferentes, otimizadas pela programação linear. Foi avaliado o desempenho da secagem através do percentual de massa acumulada no leito. Também foi realizada uma caracterização da pasta in natura e do produto final em relação à composição centesimal, solubilidade, cor e compostos fenólicos. A concentração de lipídios não influenciou o percentual de massa acumulada no leito. Porém, a pasta de vegetais com $4 \%$ de lipídios foi definida como melhor concentração, pois proporcionou melhor desempenho no equipamento e melhores características funcionais.
\end{abstract}

Palavras chave: composição, lipídios, desempenho.

\section{INTRODUÇÃO}

Estudos têm revelado que os vegetais são alimentos ricos em nutrientes e compostos antioxidantes, com destaque aos compostos fenólicos (Atoui et al., 2005). O setor agrícola destaca-se na economia brasileira através de sucessivos recordes de safras. Porém, estimase que $40 \%$ são os índices de perdas póscolheita (FAO, 2011).
Além do setor agrícola, o Brasil se destaca também, como maior exportador de carne bovina. No entanto, a indústria de carne é uma das responsáveis pelos principais impactos ambientais ligados ao alto consumo de água, alto consumo de energia e geração de efluentes líquidos, com elevada carga poluidora, como o sangue (Pacheco, 2006).

A secagem de alimentos de origem vegetal é uma prática importante, utilizada 
principalmente com objetivo de reduzir o teor de umidade, aumentando o tempo de conservação dos mesmos, e com isso melhorando o aproveitamento da produção agrícola (Oliveira e Freitas, 2004). O leito de jorro apresenta vantagens por se tratar de um equipamento de secagem com baixo custo operacional, sendo utilizado para produção em pequena escala. Apesar de produzir um pó com características similares aos secadores do tipo spray, possui algumas desvantagens na secagem de pastas e suspensões.

Dependendo da composição da pasta podem ocorrer problemas de acúmulo de material no leito, instabilidade e baixa capacidade de produção. Estudos realizados por Medeiros et al., (2004) com polpas de frutas relatam que altas concentrações de açúcares podem provocar maior aderência entre as partículas inertes. Porém, ao adicionar uma fonte lipídica melhora o desempenho do secador.

Diante disso, este trabalho teve como objetivo avaliar diferentes concentrações lipídicas a fim de melhorar o desempenho da secagem em leito de jorro de pasta de vegetais enriquecida com sangue bovino e, avaliar as características do produto desidratado.

\section{MATERIAL E MÉTODOS}

\section{Matéria-prima}

A pasta de vegetais foi constituída por: cebola branca (Allium cepa L.), tomate longa vida (Lycopersicum esculentum), cenoura (Daucus carota L.), couve (Brassica oleracea), batata (Solanum tuberosum), óleo de soja comercial e sangue bovino. A escolha dos vegetais foi feita segundo Larrosa (2012), adquiridos no comércio local da cidade do Rio Grande-RS, assim como o óleo de soja utilizado. $\mathrm{O}$ sangue bovino foi coletado em um abatedouro situado na cidade de Pelotas-RS, com as práticas de assepsia devidamente empregadas (Massaro e Pinto, 2002).

\section{Formulação da pasta}

Foram realiadas duas formulações de pasta de vegetais utilizando a programação linear (Larrosa, 2012), maximizando o valor proteico, com restrições de carboidratos (50 a $70 \%$ ), proteínas (10 a 20\%), valor calórico
(200 a 500 kcal) e fixando os valores de lipídios em 4 e 6\%. Essas concentrações foram definidas por testes preliminares, sendo que concentrações inferiores a $4 \%$ apresentou colapso do leito. A ferramenta utilizada na programação foi o aplicativo solver do MS Excel.

O processo de obtenção da pasta foi realizado no Laboratório de Operações Unitárias da FURG, conforme o fluxograma apresentado na Figura 1.

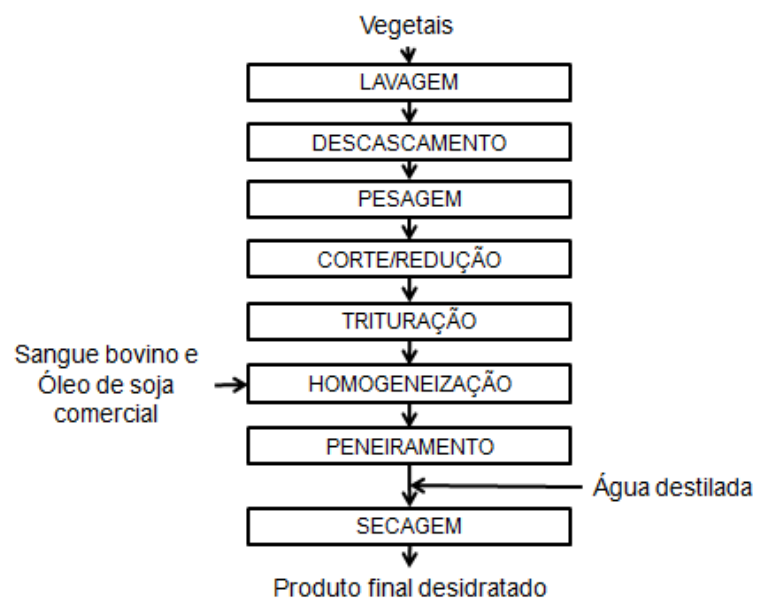

Figura 1- Fluxograma de obtenção da pasta

\section{Metodologia de secagem}

A secagem da pasta foi realizada em um secador de leito de jorro cônico que apresenta diâmetro da célula (Dc) de $17,5 \mathrm{~cm}$, com bases inferior e superior de vidro com ângulo incluso de $60^{\circ}$ e altura de $15 \mathrm{~cm}$ cada. $\mathrm{O}$ diâmetro de entrada (Di) é de $2,9 \mathrm{~cm}$, sendo a relação (Dc/ Di) igual a 6,0. O leito de partículas de inertes foi constituído por partículas de polietileno (diâmetro médio de 3,2 mm, esfericidade 0,7 e densidade de 0,96) com uma carga de $0,5 \mathrm{~kg}$. A Figura 2 apresenta um esquema do equipamento de secagem em leito de jorro. 


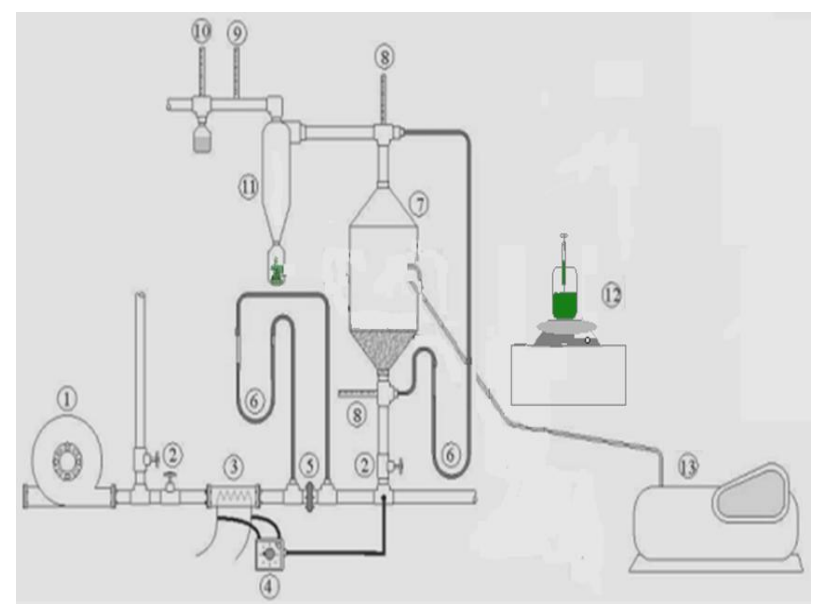

\section{Figura 1- Esquema do equipamento de secagem em leito de jorro.}

Legenda: (1) soprador radial; (2) válvulas de regulagem de vazão de ar; (3) sistema de aquecimento do ar constituído de três resistências independentes de aquecedores do tipo quartzo; (4) controlador de temperatura; (5) placa de orifício; (6) manômetro de tubo em U; (7) célula de secagem; (8) termopares de entrada e saída; (9) bulbo seco; (10) bulbo úmido; (11) ciclone tipo Lapple; (12) sistema de alimentação (13) compressor de ar.

As condições experimentais foram fixadas baseadas em testes preliminares. Utilizou-se $90^{\circ} \mathrm{C}$ como temperatura de entrada do ar e $150 \mathrm{~mL} / \mathrm{h}$ para a vazão de alimentação, A pasta foi atomizada a uma pressão de 200 $\mathrm{kPa}$ abs, com concentração de $10 \%$ de sólidos, taxa de circulação de sólidos a $100 \%$ da velocidade de jorro mínimo e um período de 4 $\mathrm{h}$ de alimentação.

O percentual de retenção de sólidos no leito foi calculado utilizando a Equação 1.

$$
\% \mathrm{R}=\frac{(\mathrm{Mf}-\mathrm{Mi}) \times(1-\mathrm{Up})}{\mathrm{Ma}(1-\mathrm{Upa})} \times 100
$$

Onde Mf é a massa final de inertes (g); Mi é a massa inicial de inertes (g); Up é a umidade do pó aderido no inerte $\left(\mathrm{g} .100 \mathrm{~g}^{-1}\right)$; Ma é a massa de pasta alimentada (g) e; Upa é a umidade da pasta alimentada $\left(\mathrm{g} \cdot 100 \mathrm{~g}^{-1}\right)$.

\section{Metodologia analítica}

Composição centesimal: A umidade foi determinada segundo a metodologia $\mathrm{n}^{\circ}$ 013/IV utilizando um sistema a vácuo à $100 \mathrm{mmHg}$ e $70^{\circ} \mathrm{C}$ (IAL, 2004). Cinzas foram determinadas pela metodologia $\mathrm{n}^{\circ} 923.03$ da AOAC (1995). As proteínas foram determinadas pelo método de micro Kjeldahl n 960.20 da AOAC, 1995. Os lipídios foram determinados pelo método de extração com mistura de solventes, segundo Bligh e Dyer (1959). E os carboidratos foram quantificados por diferença.

Compostos fenólicos: Para a determinação dos compostos fenólicos totais foram obtidos extratos fenólicos das amostras segundo a metodologia de Oliveira et al., (2007). A quantificação foi realizada pelo método espectrofotométrico (Bioespectro SP22, Brasil) utilizando o reagente FolinCiocalteau, no comprimento de onda de $750 \mathrm{~nm}$, empregando-se uma curva padrão de ácido gálico (0 a $\left.20 \mu \mathrm{g} \mathrm{mL}{ }^{-1}\right)$.

Solubilidade do pó: A solubilidade do pó foi determinada utilizando o método de Anderson et al., (1969).

Parâmetros de cor: A cor foi avaliada utilizando um sistema Minolta (CR-300, Osaka, Japão) através da medida do diagrama tridimensional de cores $\left(\mathrm{L}^{*}-\mathrm{a} *-\mathrm{b}\right)$ (Srinivasa et al., 2004). $\mathrm{O}$ ângulo $\mathrm{H}_{\mathrm{ab}}$ foi determinado utilizando a Equação 2.

$$
\mathrm{H}_{\mathrm{ab}}=\tan ^{-1}\left(\frac{\mathrm{b}^{*}}{\mathrm{a}^{*}}\right)
$$

Este ângulo caracteriza a região da tonalidade em que a amostra se apresenta. Sendo que, os ângulos $90^{\circ}, 180^{\circ}, 270^{\circ}$ e $360^{\circ}$ representam amarelo, verde, azul e vermelho, respectivamente.

\section{Análise estatística}

As respostas avaliadas foram analisadas através do teste de Tukey ao nível de $5 \%$ de significância $(\mathrm{p} \leq 0,05)$.

\section{RESULTADOS E DISCUSSÃO}

A Tabela 1 apresenta os resultados obtidos na otimização a formulação da pasta de vegetais enriquecida com sangue bovino pela programação linear. 
Tabela 1 - Massa dos componentes otimizada pela programação linear para as formulações com percentual de lipídios de 4

\begin{tabular}{ccc} 
& e 6\%. \\
\hline & \multicolumn{2}{c}{ Massa otimizada $(\mathrm{g})$} \\
\cline { 2 - 3 } Componentes & $4 \%$ de & $6 \%$ de \\
& lipídios & lipídios \\
\hline Cebola branca & 265,9 & 257,1 \\
Tomate & 205,9 & 232,9 \\
Cenoura & 237,5 & 228,2 \\
Couve & 115,8 & 111,2 \\
Batata & 37,5 & 29,9 \\
Óleo de soja & 2,1 & 4,0 \\
Sangue bovino & 35,3 & 36,8 \\
\hline
\end{tabular}

Na Tabela 1 pode-se observar que dentre os constituintes, somente o tomate, o óleo de soja e o sangue bovino apresentaram um aumento da sua quantidade otimizada ao aumentar a concentração lipídica. Isso pode ser explicado pela sua composição química, na qual representam os constituintes que contém maior percentual de lipídios.

A Tabela 2 apresenta a composição proximal em base seca para as duas pastas de vegetais e seus respectivos valores calóricos.

Tabela 2 - Comparação do percentual de constituintes e valor calórico da pasta de vegetais obtidos analiticamente para as duas concentrações de lipídios.

\begin{tabular}{ccc}
\hline Constituintes & $\begin{array}{c}\text { Concentração } \\
\text { de lipídios } \\
4 \% \%^{*}\end{array}$ & $\begin{array}{c}\text { Concentração } \\
\text { de lipídios } \\
6 \%{ }^{*}\end{array}$ \\
\hline $\begin{array}{c}\text { Proteínas } \\
(\%)\end{array}$ & $27,9 \pm 1,0^{\mathrm{a}}$ & $28,1 \pm 3,7^{\mathrm{a}}$ \\
$\begin{array}{c}\text { Carboidratos } \\
(\%)\end{array}$ & $61,4 \pm 0,9^{\mathrm{a}}$ & $60,7 \pm 3,8^{\mathrm{a}}$ \\
Lipídios (\%) & $3,9 \pm 0,1^{\mathrm{a}}$ & $5,2 \pm 0,0^{\mathrm{b}}$ \\
Cinzas (\%) & $6,8 \pm 0,1^{\mathrm{a}}$ & $6,0 \pm 0,1^{\mathrm{b}}$ \\
\hline $\begin{array}{c}\text { Valor } \\
\text { calórico (cal) }\end{array}$ & $392,5 \pm 0,7^{\mathrm{a}}$ & $419,0 \pm 10,1^{\mathrm{b}}$ \\
\hline
\end{tabular}

*Média \pm erro padrão. Letras iguais na mesma linha não apresentam diferença significativa $(p>0,05)$.

Analisando a Tabela 2, observa-se que o percentual de lipídios apresentou diferença significativa entre as duas pastas, sendo que a pasta com $4 \%$ de lipídios apresentou o valor analítico mais próximo. Por consequência da concentração de lipídios, o valor calórico também apresentou diferença significativa, sendo superior para a pasta com $6 \%$ de lipídios.

Quanto aos valores de acúmulo de sólidos no leito, não houve diferença significativa para as pastas contendo $4 \%$ $\left(23,0 \pm 2,0\right.$ g. $\left.100 \mathrm{~g}^{-1}\right)$ de lipídios e $6 \%$ de lipídios $\left(24,9 \pm 2,0\right.$ g. $\left.100 \mathrm{~g}^{-1}\right)$. Estes resultados estão de acordo com a literatura (Souza e Oliveira, 2009; Dotto et al., 2011). Apesar de não haver diferença significativa, a pasta com $4 \%$ de concentração de lipídios ao ser alimentada no leito de jorro, apresentou melhor escoabilidade das partículas inertes.

A Tabela 3 apresenta os valores de solubilidade em água e compostos fenólicos das amostras seca e in natura.

Tabela 3 - Solubilidade do produto em água e compostos fenólicos para o produto seco e à pasta in natura.

\begin{tabular}{ccc}
\hline Amostra & $\begin{array}{c}\text { Solubilidade } \\
\text { do pó* } \\
(\%)\end{array}$ & $\begin{array}{c}\text { Compostos } \\
\text { fenólicos* }^{*} \\
\left(\mathrm{mg}_{\text {EAG }} \mathrm{g}_{\text {amostra }}{ }^{-1}\right)\end{array}$ \\
\hline $4 \%$ seca & $32,7 \pm 0,7^{\mathrm{a}}$ & $12,2 \pm 0,6^{\mathrm{a}}$ \\
$6 \%$ seca & $27,6 \pm 0,8$ & $11,7 \pm 0,5^{\mathrm{a}}$ \\
$4 \%$ in natura & - & $12,2 \pm 0,3^{\mathrm{a}}$ \\
$6 \%$ in natura & - & $9,4 \pm 0,6^{\mathrm{b}}$ \\
\hline
\end{tabular}

*Média \pm erro padrão. Letras iguais na mesma coluna não apresentam diferença significativa $(\mathrm{p}>0,05)$.

Os resultados da Tabela 3 mostram que a solubilidade do pó oriundo da pasta com $4 \%$ de lipídios apresentou maior solubilidade em água. Além disso, os valores obtidos foram superiores aos encontrados na literatura, como no pó de tomate (Sousa et al., 2008) e inferiores obtidos por polpa de frutas (Dantas, et al., 2009). A solubilidade do pó irá depender das proporções de componentes hidrossolúveis como a pectina (presente no tomate), amido (presente na batata) e concentração de açúcares. Também cabe ressaltar que a presença de fibras insolúveis presentes na couve, por exemplo, pode ter dificultado a interação com a água, além de algumas substâncias lipossolúveis como os carotenoides.

Quanto aos compostos fenólicos, apenas a amostra in natura com $6 \%$ de lipídios 
apresentou diferença significativa, sendo que após a secagem o produto apresentou um aumento nos compostos fenólicos. Esse acréscimo pode estar relacionado com os compostos liberados durante a reação de Maillard, a qual ocorre devido à alta temperatura de secagem (Anese et al., 1999).

A Tabela 4 apresenta os valores de ângulo $\mathrm{H}_{\mathrm{ab}}$ do produto seco e da pasta in natura.

Tabela 4 - Ângulo $H_{a b}$ do produto seco e da pasta in natura.

\begin{tabular}{cc}
\hline $\begin{array}{c}\text { Concentração de } \\
\text { lipídios }\end{array}$ & $\mathrm{H}_{\mathrm{ab}}{ }^{*}\left(^{\circ}\right)$ \\
\hline $4 \%$ seca & $86,6 \pm 0,1^{\mathrm{a}}{ }^{\mathrm{a}}$ \\
$6 \%$ seca & $86,5 \pm 0,1^{\mathrm{b}}$ \\
$4 \%$ in natura & $102,0 \pm 0,1^{\mathrm{c}}$ \\
$6 \%$ in natura & $91,8 \pm 0,2^{\mathrm{c}}$ \\
\hline
\end{tabular}

*Média \pm erro padrão. Letras iguais na mesma coluna não apresentam diferença significativa $(\mathrm{p}>0.05)$.

$\mathrm{Na}$ Tabela 4, os valores de ângulo $\mathrm{H}_{\mathrm{ab}}$ não apresentaram diferença significativa entre as duas amostras secas. No entanto, as duas diferenciaram-se significativamente em relação a sua pasta de origem ao nível de 5\% de significância $(\mathrm{p}<0,05)$. Sendo que, a pasta in natura apresentou ângulos localizados no segundo quadrante do diagrama tridimensional de cores com coloração amarelo-esverdeado e posteriormente a secagem desta pasta, os ângulos se localizaram no primeiro quadrante, com coloração amarelo-avermelhado.

\section{CONCLUSÃO}

A concentração de lipídios não influenciou o percentual de massa acumulada de sólidos no leito. No entanto, a pasta de vegetais com $4 \%$ de lipídios foi definida como a mais adequada para a secagem em leito de jorro, pois esta proporcionou melhor desempenho no equipamento. Além disso, a pasta com $4 \%$ de lipídios apresentou um produto de maior qualidade quanto à solubilidade do pó $(32,7 \pm 0,7 \%)$ e compostos fenólicos $\left(12,2 \pm 0,6 \mathrm{mg}_{\mathrm{EAG}} \mathrm{g}_{\text {amostra }}{ }^{-1)}\right.$.

\section{REFERÊNCIAS}

ANESE, M.; MANZOCCO, L.; NICOLI, M.C.; LERICI, C.R. (1999), Antioxidant properties of tomato juice as affected by heating. Journal of the Science of Food and Agriculture, v. 79, p. 750-754.

ANDERSON, R.A.; CONWAY, H.F.; PFEIFER, U.F.; GRIFFIN JR., E.L.(1969), Gelatinization of corn grits by roll and extrusion cooking. Cereal Science Today, Minnesota, v. 14, n. 1, p. 4-7.

A.O.A.C. (1995), Association of Official Analytical Chemists. Official Methods of Analysis, 16th edn. Arlington, Virginia, v. 1.

ATOUI, A. K.; MANSOURI, A.; BOSKOU, G.; KEFALAS, P. (2005), Tea and herbal infusions: their antioxidant activity and phenolic profile." Food Chem., v. 89, p. 27-36.

BLIGH, E. G.; DYER, W. J. (1959), A rapid method for total lipid extraction and purification. Canadian Journal of Biochemistry and Physiology, 37, p. 911917.

DANTAS, T. N. P.; SOUZA, J. S.; JÚNIOR, F. E. S.; MEDEIROS, M. F. D. (2009), Propriedades físicas e físico-químicas de pós de misturas de polpas de frutas com diferentes fontes lipídicas. VIII Congresso Brasileiro de Engenharia Química em Iniciação Científica, Uberlândia, MG.

DOTTO, G. L., SOUZA, V. C., \& PINTO, L. A. A. (2011), Drying of chitosan in a spouted bed: The influences of temperature and equipment geometry in powder quality. LWT - Food Science and Technology, 44, 8, 1786-1792.

FOOD AND AGRICULTURE ORGANIZATION. FAO. (2011), Global food losses and food waste. International Congress Save Food. Rome.

INSTITUTO ADOLFO LUTZ. (2004), Métodos físico-químicos para análise de alimentos. 4. ed. São Paulo, 1032 p.

LARROSA, A. P. Q. (2012), Secagem de pasta de vegetais: análise da operação em leito de jorro e caracterização do produto final. Rio Grande: FURG, 129 p. Dissertação. (Mestrado em Engenharia e Ciência de Alimentos). 
MASSARO, A.; PINTO, L. A. A. (2002), Enriquecimento proteico de farelo de arroz desengordurado com sangue bovino, utilizando a técnica de leito de jorro." Revista do Instituto Adolfo Lutz, v. 61, n. 2, p. 77-84.

MEDEIROS, M. F. D.; ALSINA, O. L. S.; ROCHA, S. C. S.; JERÔNIMO, C. E. M.; MEDEIROS, U. K. L.(2004), Drying of pastes in spouted beds: influence of the paste composition on the material retention in the bed. Drying-Proceedings of the $14^{\text {th }}$ Internationl Drying Symposium-IDS, p. 1529-1536.

OLIVEIRA, H. V. A.; FREITAS, L. A. P. (2004), The effect of processing factors on the efficiency of hard gelatin capsules coating in a spouted bed. 14th International Drying Symposium. v. B, p. 852 - 859, São Paulo.

OLIVEIRA, M. S.; DORS, G. C.; SOUZASOARES, L. A.; BADIALE-FURLONG, E. (2007), Antifungal and antioxidant activity of vegetables extracts." Food and Nutrition, v. 18, p. 267-275.

PACHECO, J. W. (2006), Guia técnico ambiental de abates (bovino e suíno). São Paulo: CETESB.

SOUSA, A. S. S.; BORGES, S. V.; MAGALHÃES, N. F.; RICARDO, H. V.; AZEVEDO, A. D. (2008), Spray-dried tomato powder: reconstitution properties and colour. Brazilian Archives of Biology and Technology, 51, n. 4, p. 807-814.

SOUZA, C. R. F.; OLIVEIRA, W. P. (2009), Drying of herbal extract in a draft-tube spouted bed. The Canadian Journal of Chemical Engineering, 87, p. 279-288.

SRINIVASA， P. C.; RAMESH， M. N.; KUMAR, K. R.; THARANATHAN, R. N. (2004), Properties of chitosan films prepared under different drying conditions. Journal of Food Engineering. v. 63, p. $79-85$.

\section{AGRADECIMENTOS}

Os autores agradecem ao CNPq, Capes, Programa de Educação Tutorial e a FURG pelo apoio financeiro. 